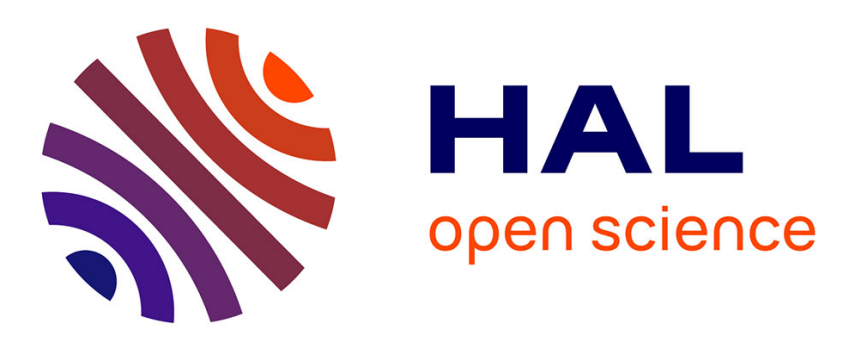

\title{
GPS/INS/optic flow data fusion for position and Velocity estimation
}

Diego Mercado, Gerardo Ramon Flores Colunga, Pedro Castillo, Juan

Antonio Escareño, Rogelio Lozano

\section{- To cite this version:}

Diego Mercado, Gerardo Ramon Flores Colunga, Pedro Castillo, Juan Antonio Escareño, Rogelio Lozano. GPS/INS/optic flow data fusion for position and Velocity estimation. International Conference on Unmanned Aircraft Systems (ICUAS 2013), May 2013, Atlanta, Georgia, United States. pp.486 - 491. hal-00939204

\section{HAL Id: hal-00939204 https://hal.science/hal-00939204}

Submitted on 30 Jan 2014

HAL is a multi-disciplinary open access archive for the deposit and dissemination of scientific research documents, whether they are published or not. The documents may come from teaching and research institutions in France or abroad, or from public or private research centers.
L'archive ouverte pluridisciplinaire HAL, est destinée au dépôt et à la diffusion de documents scientifiques de niveau recherche, publiés ou non, émanant des établissements d'enseignement et de recherche français ou étrangers, des laboratoires publics ou privés. 


\title{
GPS/INS/Optic Flow Data Fusion for Position and Velocity estimation*
}

\author{
D. A. Mercado ${ }^{1}$, G. Flores ${ }^{1}$, P. Castillo ${ }^{1,2}$, J. Escareno ${ }^{3}$ and R. Lozano ${ }^{1,2}$
}

\begin{abstract}
This paper presents a simple and easy to implement sensor data fusion algorithm, using a Kalman filter (KF) in a loosely coupled scheme, for estimation of the velocity and position of an object evolving in a three dimensional space. A global positioning system (GPS) provides the position measurement while the velocity measurement is taken from the optical flow sensor, finally, the inertial navigation system (INS) gives the acceleration, which is considered as the input of the system. Real time experimental results are shown to validate the proposed algorithm.
\end{abstract}

\section{INTRODUCTION}

Unmanned aerial vehicles (UAVs) have become important tools for exploration, surveillance, search and rescue applications thanks to the great advances in sensors manufacturing which permit improving the accuracy of the measurements, miniaturization and cost reduction. However, a precise knowledge of the position and velocity of the UAVs for outside hover flight position control applications is still a big challenge. Not expensive GPS sensors can provide this information, however, the errors, of $2 \mathrm{~m}$ at best, are not suitable for precise applications, neither their low measurement rate of about 5Hz. Even more, GPS signal can be easily lost leaving the system without a position measurement. Another alternative widely studied are the optical flow sensors which use vision algorithms for estimating the motion velocity of a system. Nevertheless they are sensible for lighting changes.

Several related works to data fusion for UAV loaclization can be found in the literature, however, the most of them use post-processed data took from a fast moving object, while for this work, real time experiments for static objects are also of interest, since that is the case for an helicopter in hover flight. For example, in [1] several GPS/INS fusion algorithms are presented using both, extended Kalman filter (EKF) and unscented Kalman filter (UKF) in a loosely coupled scheme for attitude estimation and validated in experiments with post-processed data. Similarly in [2], the authors present an attitude estimation algorithm with data from GPS and INS sensors using a KF. A tightly coupled EKF scheme is employed in [3], for delivering all available information from the satellites to an INS/GPS sensor fusion algorithm,

\footnotetext{
*This work was supported by project SEARCH and the Institute for Science \& Technology of Mexico City (ICyTDF)

${ }^{1}$ HEUDIASYC UMR 7253 CNRS-UTC, Compiègne, France. dmercado, floresc, rlozano, pedro.castillo@hds.utc.fr

${ }^{2}$ LAFMIA UMI 3175 CINVESTAV-CNRS, Mexico.

3 Automatic Control and Micro-Mechatronic Systems (AS2M) department at FEMTO-ST Institute, Besanon - France.
}

even when only one satellite is observable (traditional loosely coupled filters require at least four observed satellites to work properly). Additionally, an EKF with covariance matching based adaptation algorithm is implemented in [4] using GPS, INS and optical flow measurements. In [5] two KFs are used to fusion data from GPS, INS, computer vision and a laser range finder. Sensor fusion using Kalman filters is also widely used for improving data obtained from computer vision. For example, in [6] an EKF is presented for target tracking and optical flow navigation in a GPS denied environment using computer vision and INS data, while in [7] different optical flow algorithms are integrated by a KF for a better estimation of velocity. A different approach is shown in [8] where using multiple UAVs, the position of a particular UAV can be estimated when the GPS signal has been lost, by measuring the distance with respect to the other UAVs and employing an EKF. These positioning strategies have also a great potential of application outside the mobile robotics field, for example, in [9] they are used for aiding blind pedestrian positioning.

The aim of this work is to take information from multiple different kinds of sensors in order improve data and have a precise and reliable estimation of the position and velocity. In order to do so, the use of a $\mathrm{KF}$ is explored in a loosely coupled scheme for fusing the information obtained from GPS, INS and optical flow sensors.

This work is organized as follows: In section II, the problem of interest is settled, as well as a reminder of the basic Kalman filter's equations to be used to solve it. In section III, the data fusion formulation is presented together with the optic flow algorithm. Experimental platform is described and results are shown in section IV. Finally, conclusions are made and future work is established in section V.

\section{PROBLEM STATEMENT}

A low-cost GPS unit generally provides an estimate of the position within a few meters, by means of an internal Kalman filter. However, such GPS estimation is likely to be noisy; readings vary rapidly, though always remaining within a few meters of the real position. This inaccurate position estimation is not enough to accomplish the high demands required in emerging micro and mini aerial vehicles (MAV) applications [10], [11]. This estimation gives an error depending on some surrounding conditions; like number and geometry of satellites in view, the quality of GPS receiver or the presence of objects that interfere with the signals from the satellites [14]. An estimate for the accuracy is provided in the GPS data-stream and is called accP for the position and $a c c V$ for the velocity. This accuracy changes dynamically 
depending on the actual conditions and represents an estimate of the error's magnitude, or in other words, the reliability of the measurement.

Therefore, the idea is to provide extra information from other sensors to improve the estimate of the position and velocity, especially when the accuracy value of the GPS tends to be too big. Let us consider a Kalman filter.

\section{A. Discrete Kalman Filter}

The Kalman filter is a well know, widely used optimal state estimator [12], [13] of a discrete time linear dynamic system perturbed by white noise

$$
\begin{gathered}
\xi_{k}=A \xi_{k-1}+B u_{k-1}+\omega_{k-1} \\
Z_{k}=H \xi_{k}+v_{k}
\end{gathered}
$$

considering the state vector $\xi$, the input vector $u$ and the measurement vector $Z$. $A, B$ and $H$ stand for the state transition, input and observation matrices, respectively, and subindex $k$ is the discrete time index. The process and measurement noise $\omega, v$ are supposed to be white with normal probability distributions, i.e.

$$
\begin{gathered}
\omega \sim N(0, Q) \\
v \sim N(0, R)
\end{gathered}
$$

with $Q, R$ being the process noise covariance and the measurement noise covariance, respectively.

Then, the a priori state estimates $\hat{\xi}_{k}^{-}$is given by

$$
\hat{\xi}_{k}^{-}=A \hat{\xi}_{k-1}+B u_{k-1}
$$

the a priory error covariance $P_{k}^{-}$can be calculated as

$$
P_{k}^{-}=A P_{k-1} A^{T}+Q
$$

Now, the Kalman gain matrix $K_{k}$ can be determined

$$
K_{k}=P_{k}^{-} H^{T}\left(H P_{k}^{-} H^{T}+R\right)^{-1}
$$

and after measuring the process, the a posteriori state estimate $\hat{\xi}$ and error covariance $P_{k}$ are respectively

$$
\begin{gathered}
\hat{\xi}_{k}=\hat{\xi}_{k}^{-}+K_{k}\left(Z_{k}-H \hat{\xi}_{k}^{-}\right) \\
P_{k}=\left(I-K_{k} H\right) P_{k}^{-}
\end{gathered}
$$

\section{GPS/INS/OPTIC FLOW DATA FUSION}

\section{A. System Model}

Consider the state vector

$$
\xi=\left[\begin{array}{llllll}
x & y & z & V x & V y & V z
\end{array}\right]^{T}
$$

which represents the position $(x, y, z)$ and velocity $(V x, V y, V z)$ of an object moving in the three dimensional space, with respect to the inertial frame $I$ fixed to the ground. Thus, the motion equations of the system can be written as

$$
\dot{\xi}=\left[\begin{array}{c}
\dot{x} \\
\dot{y} \\
\dot{z} \\
\dot{V}_{x} \\
\dot{V}_{y} \\
\dot{V}_{z}
\end{array}\right]+\omega=\left[\begin{array}{c}
V x \\
V y \\
V z \\
u
\end{array}\right]+\omega
$$

where $u=\left[\begin{array}{lll}a_{x} & a_{y} & a_{z}\end{array}\right]^{T}$ are the inputs of the system. In our case the accelerations measured from the INS sensor, and $\omega \in \mathfrak{R}^{6}$ stands for the process noise. Then the measurement vector is given by

$$
Z=\left[\begin{array}{c}
x_{G P S} \\
y_{G P S} \\
z_{G P S} \\
V_{O F_{x}} \\
V_{O F_{y}} \\
V z_{h}
\end{array}\right]=\left[\begin{array}{c}
x \\
y \\
z \\
V x \\
V y \\
V z
\end{array}\right]+v
$$

with $\left[\begin{array}{lll}x_{G P S} & y_{G P S} & z_{G P S}\end{array}\right]^{T}$ being the position computed from the GPS sensor, $V_{O F_{x}}$ and $V_{O F_{y}}$ define the translational velocities in the horizontal plane calculated from an optical flow algorithm, and $V z_{h}$ denotes the vertical velocity. $v \in \mathfrak{R}^{6}$ represents the measurement noise.

In order to implement the KF, the system (11) has to be discretized. By means of the Euler's forward method one gets

$$
\xi_{k}=\left[\begin{array}{c}
x_{k-1}+T V x_{k-1} \\
y_{k-1}+T V y_{k-1} \\
z_{k-1}+T V z_{k-1} \\
V x_{k-1}+T a_{x_{k-1}} \\
V y_{k-1}+T a_{y_{k-1}} \\
V z_{k-1}+T a_{z_{k-1}}
\end{array}\right]+\omega_{k-1}
$$

where $T$ defines the sampling period.

\section{B. Optical Flow}

In this study we consider the camera-experimental platform arrangement moving in a 3-dimensional space with respect to a rigid scene. The camera velocities in the inertial frame are given by $\left(V_{x}, V_{y}, V_{z}\right)$. The optical flow computed at an image point $\left(x_{i}, y_{i}\right)$ is composed of a translational and rotational part as follows [15]

$$
\left[\begin{array}{l}
O F_{x i} \\
O F_{y i}
\end{array}\right]=T_{O F}+R_{O F}
$$

where the rotational part $R_{O F}$ is given as

$$
R_{O F}=\left[\begin{array}{ccc}
\frac{x_{i} y_{i}}{f_{x}} & -\left(f_{x}+\frac{\left(x_{i}\right)^{2}}{f_{x}}\right) & y_{i} \\
\left(f_{y}+\frac{\left(y_{i}\right)^{2}}{f_{y}}\right) & -\frac{x_{i} y_{i}}{f_{y}} & -x_{i}
\end{array}\right]\left[\begin{array}{c}
\omega_{\mathrm{x}} \\
\omega_{\mathrm{y}} \\
\omega_{\mathrm{z}}
\end{array}\right]
$$

and the translational part as

$$
T_{O F}=\frac{1}{z}\left[\begin{array}{ccc}
-f_{x} & 0 & x_{i} \\
0 & -f_{y} & y_{i}
\end{array}\right]\left[\begin{array}{l}
\dot{x}^{c} \\
\dot{y}^{c} \\
\dot{z}^{c}
\end{array}\right]
$$

From (15), $O F_{x i}$ and $O F_{y i}$ are the optical flow components in the $x$ and $y$ coordinates, respectively, of the $\left(x_{i}, y_{i}\right)$ feature. $\left(\dot{x}^{c}, \dot{y}^{c}, \dot{z}^{c}\right)$, in (17), are the camera translational velocities and $\left(w_{x}, w_{y}, w_{z}\right)$ are the camera rotation rates. The focal lengths of the camera are $f_{x}$ and $f_{y}$.

The optical flow is computed by using the pyramidal implementation of the Lucas-Kanade algorithm [16]. With the 


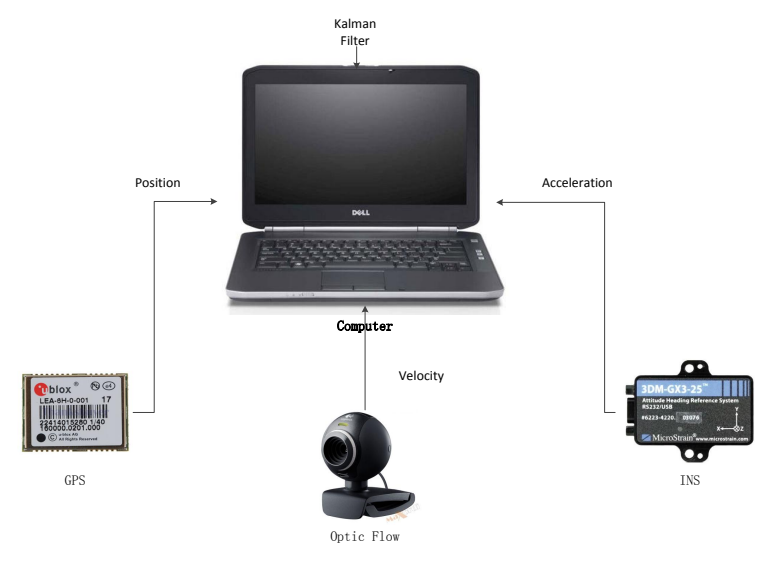

Fig. 1. Experimental platform.

purpose of obtaining a better estimation, the Lucas-Kanade algorithm is coupled with a rich texture point detector .

When computing the optical flow, all the $\left(x_{i}, y_{i}\right)$ feature's coordinates are known, furthermore, they all share the same movement. By using all the tracked features, a mean value for the optical flow can be expressed as

$$
\begin{aligned}
& \overline{O F}_{x}=\bar{V}_{O F_{\mathrm{x}}}+K_{x} \bar{V}_{O F_{\mathrm{z}}}+\bar{R}_{O F_{x}} \\
& \overline{O F}_{y}=\bar{V}_{O F_{\mathrm{y}}}+K_{y} \bar{V}_{O F_{\mathrm{z}}}+\bar{R}_{O F_{y}}
\end{aligned}
$$

where $\overline{O F}_{x}$ and $\overline{O F}_{y}$ are the means of the optical flow sensed in the image coordinate system, $\bar{V}_{O F_{\mathrm{z}}}$ represents the relative depth and $K_{x}$ and $K_{y}$ are known scale factors depending on intrinsic parameters of the camera. The rotational optical flow terms $\bar{R}_{O F_{x}}$ and $\bar{R}_{O F_{y}}$ are despicable for this study since the camera movement is fixed to the horizontal plane, otherwise, they have to be compensated. Then, the pseudospeeds $\left(\bar{V}_{O F_{x}}, \bar{V}_{O F_{y}}, \bar{V}_{O F_{z}}\right)$ can be represented as

$$
\begin{aligned}
\bar{V}_{O F_{x}} & =-f_{x} \frac{\dot{x}}{z} \\
\bar{V}_{O F_{y}} & =-f_{y} \frac{\dot{y}}{z} \\
\bar{V}_{O F_{z}} & =\frac{\dot{z}}{z}
\end{aligned}
$$

In the experimental setup, the camera is mounted on the experimental platform, thus both of them share the same translational movement, i.e, $\left(\dot{x}^{c}, \dot{y}^{c}, \dot{z}^{c}\right)=(\dot{x}, \dot{y}, \dot{z})$.

\section{EXPERIMENTAL RESULTS}

In order to validate in real time the proposed fusion schema, it was developed an experimental platform consisting of a wheeled table equipped with a GPS, an INS and a camera for the optical flow (see Fig. 1). All the algorithms are performed in a portable computer attached to the table. To simplify the experiment, only the states in the $x y$ plane are estimated, therefore, the height $z$ is fixed at a constant value. In order to extend the estimation to a 3-dimensional space, with variable height, a height sensor is required. The
OpenCV libraries are used to capture the image from the camera, perform the optical flow and estimate the horizontal velocities. Similarly, the KFilter library is used to implement the Kalman filter. A GPS provides the measurements of the position at a rate of $5 \mathrm{~Hz}$ with an error of $2 \mathrm{~m}$ in the best scenario. The vision algorithm is executed at a rate of $40 \mathrm{~Hz}$ and the INS sensor measures the accelerations using accelerometers. Notice that the developed platform can be easily used to test other data fusion configurations including nonlinear formulations.

Test results for the case when the object is fixed to a point in an outside urban environment, surrounded by buildings using only natural light (in this case a parking area with sunlight), are presented in Figures 2 - 10. The process noise matrix $Q$ is set diagonal with very little values, i.e

$$
Q=\left[\begin{array}{cccccc}
0 & 0 & 0 & 0 & 0 & 0 \\
0 & 0 & 0 & 0 & 0 & 0 \\
0 & 0 & 0 & 0 & 0 & 0 \\
0 & 0 & 0 & 0.005 & 0 & 0 \\
0 & 0 & 0 & 0 & 0.005 & 0 \\
0 & 0 & 0 & 0 & 0 & 0.005
\end{array}\right]
$$

It is hard to know the exact value for the measurement covariance matrix $R$, since the GPS data stream does not provide it, and the experimental conditions are always changing (e.g. the number of satellites in range and its position, or the lighting). However, this parameter can be used to inform the filter about the reliability of a measurement. In addition, we propose to relate the estimate accuracy parameter from the GPS ( $a c c P$ ) to matrix $R$ such that when the GPS's conditions are not appropriate, the Kalman filter stops trusting the GPS measurements and bases its estimations on the process model and the other measurements. Hence, the measurement noise covariance matrix $R$ is selected diagonal with values directly proportionals to the GPS accuracy estimation $(a c c P)$, for position measurements from the GPS and small ones for the corresponding to the optical flow velocity measurements, i.e.

$$
R=\left[\begin{array}{cccccc}
a c c P & 0 & 0 & 0 & 0 & 0 \\
0 & a c c P & 0 & 0 & 0 & 0 \\
0 & 0 & a c c P & 0 & 0 & 0 \\
0 & 0 & 0 & 0.1 & 0 & 0 \\
0 & 0 & 0 & 0 & 0.1 & 0 \\
0 & 0 & 0 & 0 & 0 & 0.1
\end{array}\right]
$$

The accelerations of the system, measured by the INS, are shown in Fig. 2. As expected, they are very close to zero since the object is not moving. Figures 3 and 4 present, respectively, the position and velocity of the object measured from the GPS sensor. As it is well known, the GPS is not reliable under these conditions (remaining in a static position, in an urban area surrounded by buildings which interfere with the satellites signals), as can be observed from Fig. 5 which displays the GPS accuracy (under ideal conditions it is supposed to be of $2 \mathrm{~m}$ ). Velocity computed from the optical flow algorithm is exhibit in Fig. 6, while the estimated estates from the Kalman filter are shown in Fig. 7 and 8 for the position and velocity, respectively. Finally, a comparison 


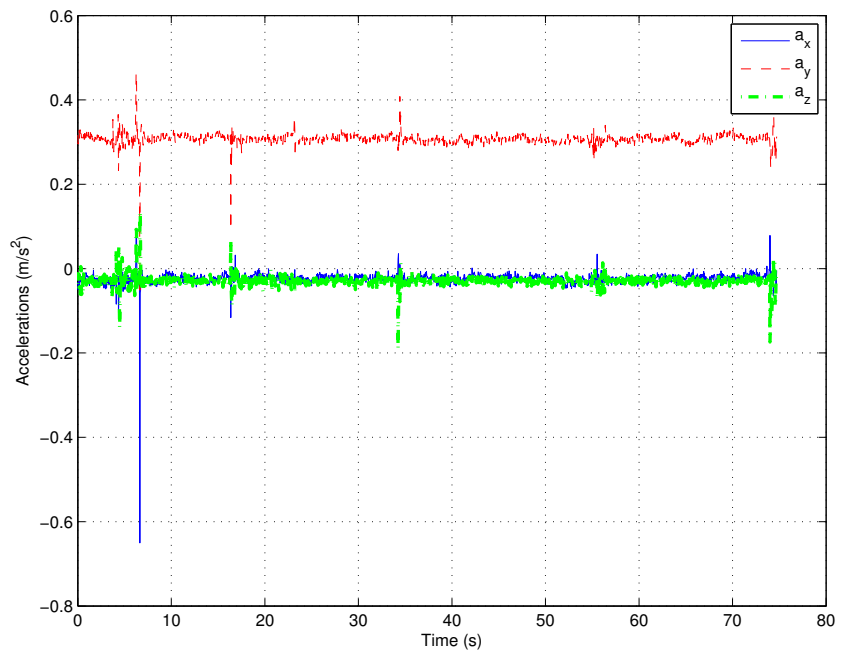

Fig. 2. Accelerations measured from the INS.

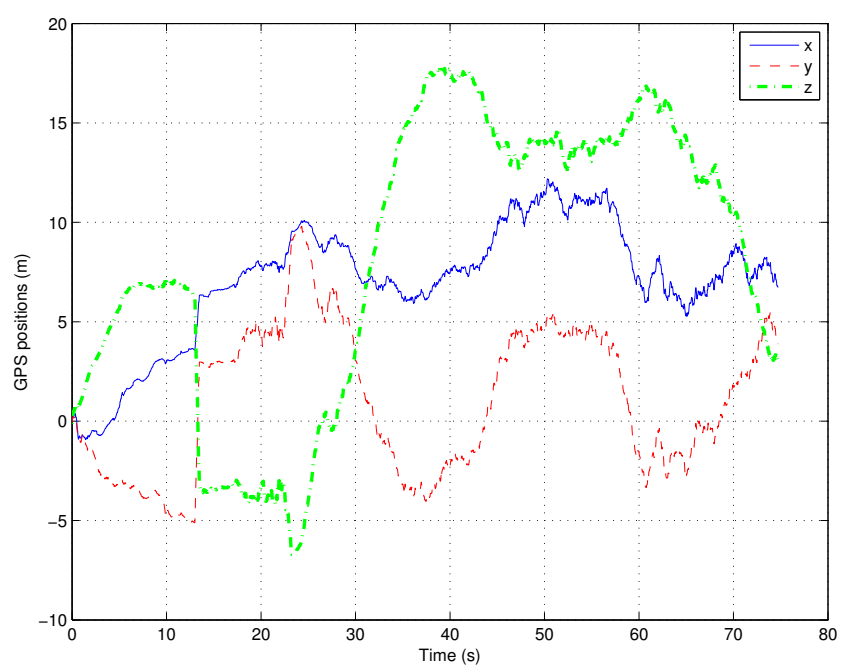

Fig. 3. GPS position.

between the position estimated only by the GPS and the one estimated using the proposed fusion algorithm is presented in Fig. 9 for the $x y$ plane and in Fig. 10 for a three dimensional space view.

\section{CONCLUSIONS AND FUTURE WORK}

In this work, a GPS/INS/Optic flow data fusion algorithm using a KF was developed and implemented, for velocity and position estimation. Experimental results were shown and, from them, it can be observed that under bad GPS conditions and a good velocity measurement from the optical flow sensor, the estimate position and velocity were improved with respect to the direct measurement from the GPS. Further experiments are required to completely validate the proposed observer scheme.

$\mathrm{KF}$ implies a tradeoff between measured data from the sensors and expected state from the dynamic model, this

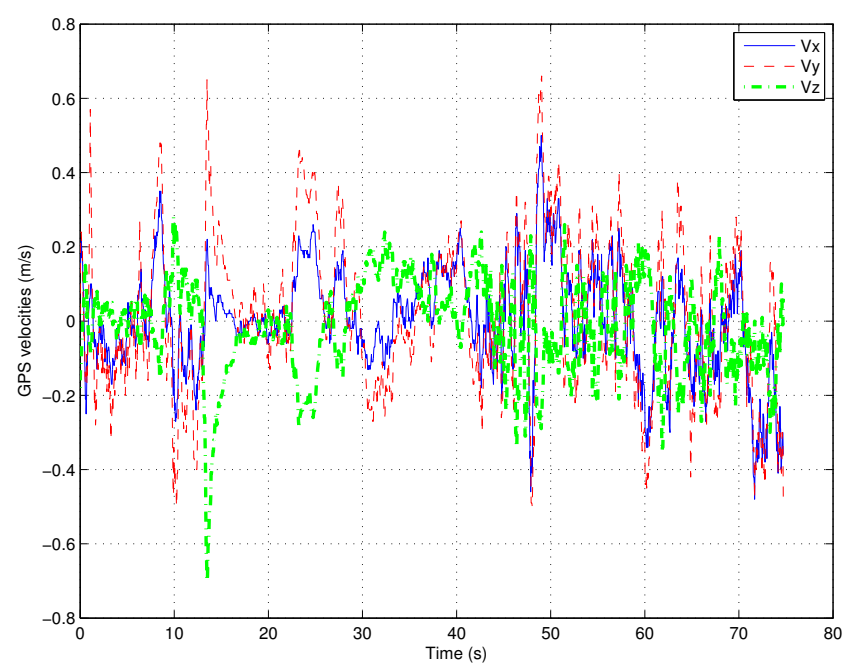

Fig. 4. GPS velocities.

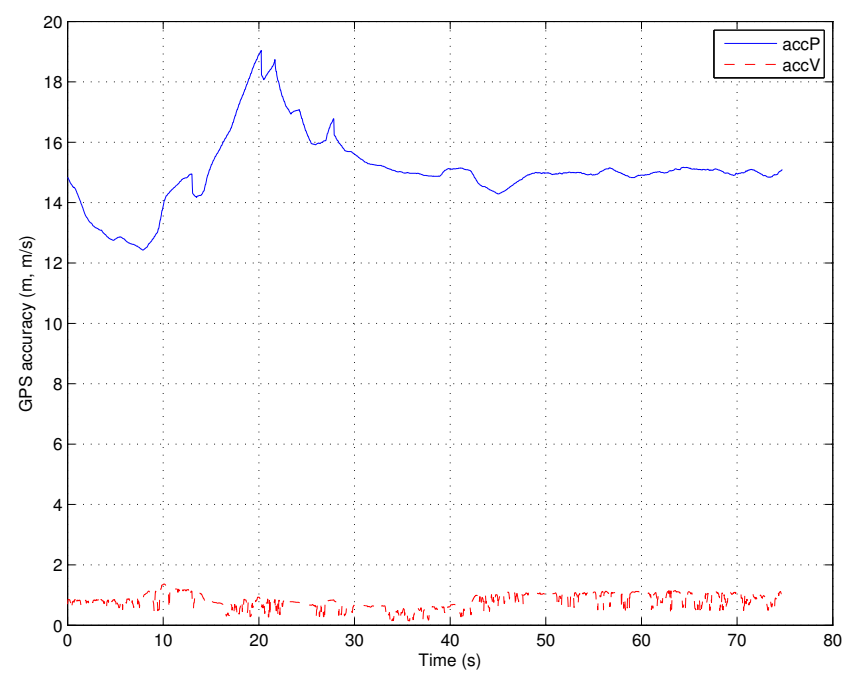

Fig. 5. GPS accuracy.

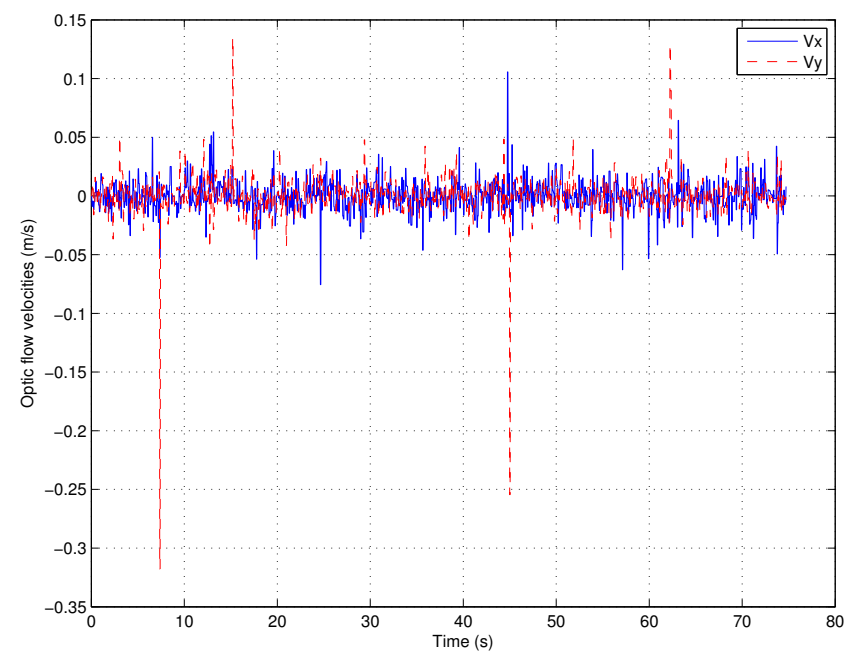

Fig. 6. Optic flow velocity. 


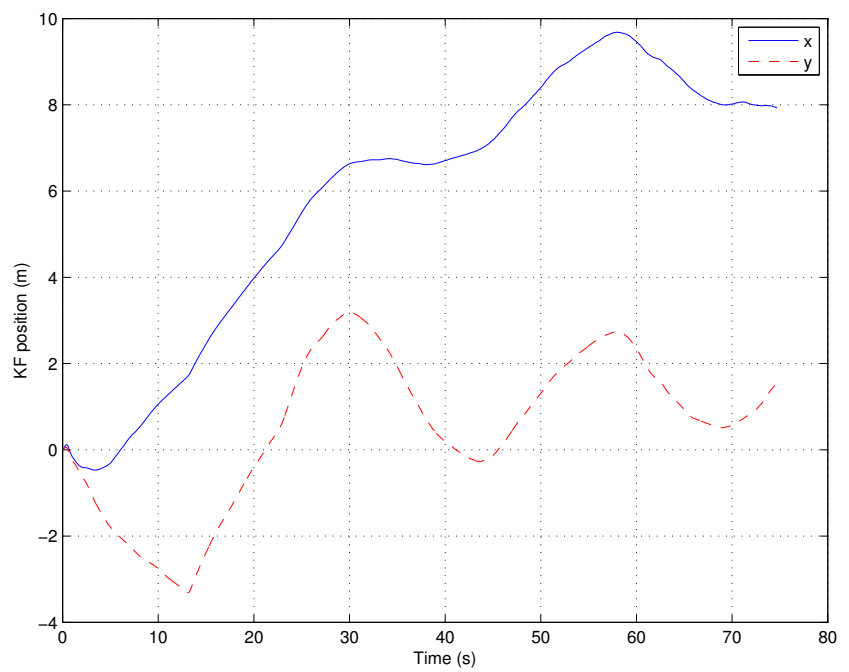

Fig. 7. KF estimate position.

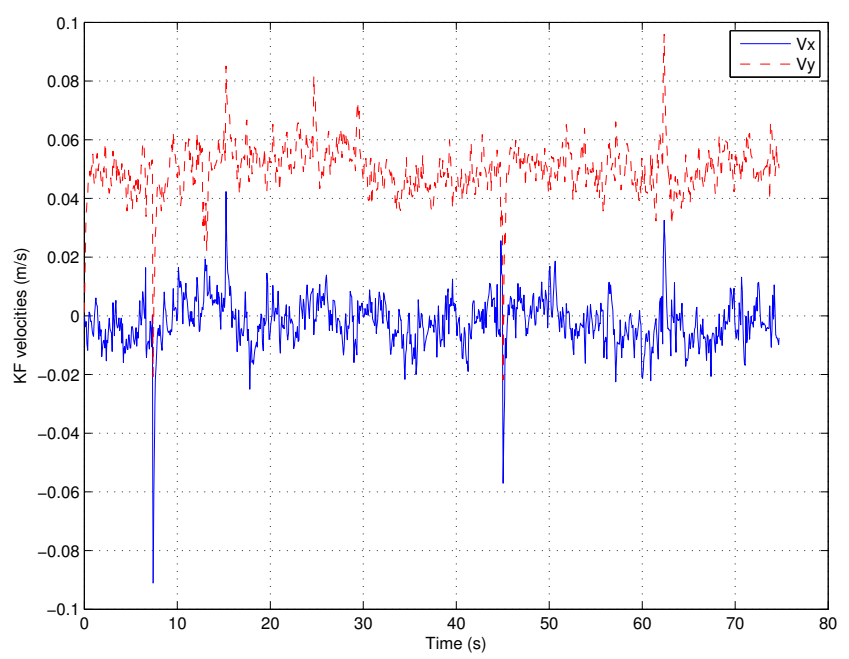

Fig. 8. KF estimate velocity.

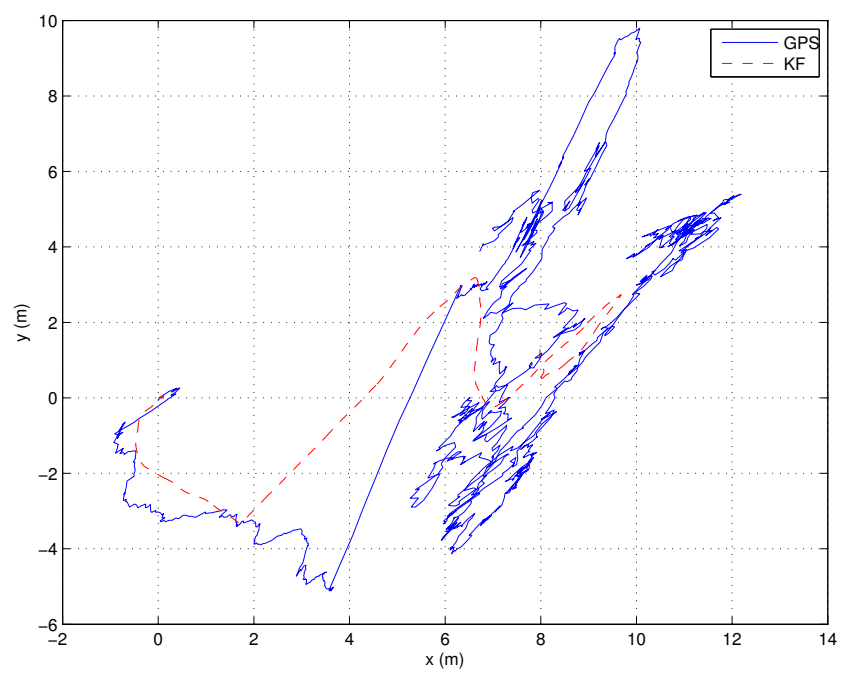

Fig. 9. GPS vs KF position.

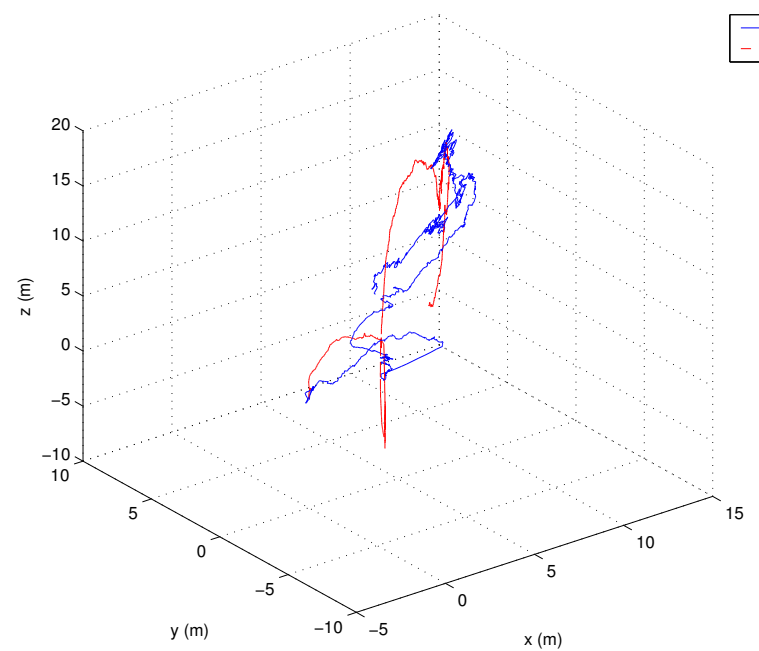

Fig. 10. GPS vs KF position space view.

tradeoff is somehow related to the process and measurement noise (bigger measurement noise implies less reliable measurements). This can be used to improve results in changing environments, where noise is hard to model, by changing dynamically the process noise covariance matrix in order to rely on the measured data when the conditions are ideal for the sensors and otherwise to rely on the process.

Future work includes implementation and analysis of other data fusion formulations with these three sensors (GPS, INS and camera) to find out the best solution for the considered problem. Also, it is highly desired to implement this data fusion algorithms embedded in an UAV for solve the position and trajectory tracking control problems for outside applications.

\section{REFERENCES}

[1] Gross, J., Gu, Y., Rhudy, M., Gururajan, S. \& Napolitano, M. Flighttest evaluation of sensor fusion algorithms for attitude estimation. In IEEE Transactions on Aerospace and Electronic Systems. vol.48, No.3, 2012.

[2] Yoo, C. \& Ahn, I. Low cost GPS/INS sensor fusion system for UAV navigation. In Digital Avionics Systems Conference, DASC '03. The 22nd, vol.2, pp 8.A.1-1- 8.A.1-9, Indianapolis, USA, 2003.

[3] George, M. \& Sukkarieh, S. Tightly coupled INS/GPS with bias estimation for UAV applications. In Proceedings of the 2005 Australian Conferences on Robotics and Automation., Sidney, Australia, 2005.

[4] Ding, W., Wang, J. \& Almagbile, A. Adaptive filter design for UAV navigation with GPS/INS/optical flow integration. International Conference on Electrical and Control Engineering, Wuhan, China, 2010.

[5] Wang, J., Garratt, M., Lambert, A., Jianguo, J., Han, S. \& Sinclair, D. Integration of GPS/INS/vision sensors to navigate unmanned aerial vehicles. In the International Archives of the Photogrammetry, Remote Sensing and Spatial Information Sciences. Vol. XXXVII. Part B1. Beijing 2008.

[6] Watanabe, Y., Fabiani, P. \& Le Besnerais, G. Simultaneous Visual Target Tracking and Navigation in a GPS-Denied Environment. In International Conference on Advanced Robotics, Munich, Germany, 2009.

[7] Song, X. \& Seneviratne, L. A Kalman Filter-Integrated Optical Flow Method for Velocity Sensing of Mobile Robots. In IEEE/ASME Transactions on Mechatronics, vol.16, No.3, 2011. 
[8] Mao, G., Drake, S. \& Anderson, B. Design of a Extended Kalman Filter for UAV Localization. In Information, Decision and Control, Adelaide, Australia, 2007.

[9] Brilhault, A., Kammoun, S., Gutierrez, O., Truillet, P. \& Jouffrais, C. Fusion of Artificial Vision and GPS to Improve Blind Pedestrian Positioning. In New Technologies, Mobility and Security (NTMS), Paris, France, 2011.

[10] Paul E. Pound, Daniel R. Bersak \& Aaron M. Dollar. Grasping From the Air: Hovering Capture and Load Stability. IEEE International Conference on Robotics and Automation, Shanghai, China, May. 2011.

[11] D. Mellinger, M. Shomin, N. Michael, \& V. Kumar. Grasping and Transport using Multiple Quadrotors, Distributed Autonomous Robotic Systems. Cooperative, Lausanne, Switzerland, Nov 2010.

[12] Grewal, M. \& Andrews, A. Kalman filtering: theory and practice using MATLAB. John Wiley \& Sons. 2001.

[13] Brown, R. \& Hwang, Y. Introduction to random signals and applied Kalman filtering, second edition, John Wiley \& Sons. 1992.

[14] Grewal, M., Weill, L. \& Andrews, A. Global positioning systems, inertial navigation, and integration. John Wiley \& Sons. 2001.

[15] Matthies, L. \& Kenade, T. Kalman Filter-based Algorithms for Estimating Depth from Image Sequences. International Journal of Computer Vision, vol.3, No.3, pp 209-236, 1989.

[16] G. Bradski \& A. Kaehler. Learning OpenCV: Computer Vision with the OpenCV Library. O'Reilly Media. 2008. 